\title{
Sexual Dysfunction in Patients With Diabetes
}

An article by $Z_{\text {weifler and his colleagues }}{ }^{1}$ in this issue of the $7 A B F P$ addresses a topic of profound importance to family practice patients. Normal expression of a person's sexuality depends on many things, most importantly his or her general health. Disease can interfere with this highly personal part of ourselves, but unlike other aspects of the human condition, even discussing this intimate aspect of our lives in the event of clear dysfunction can be a personal challenge. Indeed, as the authors point out, most men they questioned who suffered with frequent erectile dysfunction chose not to consult their physician about it. Yet, as their advocates, primary care providers are in the best position to offer sound counsel and proved therapeutic interventions.

To consider male sexual dysfunction only in such a discussion, however, is admittedly missing one half of the disease before us. Female sexual dysfunction related to diabetes, hypertension, and other illness must certainly be as common, although considerably less visible in the medical literature. Growing data support the notion that women with diabetes develop symptoms related to failure of the same autonomic pathways that have been found to mediate erectile dysfunction in men. ${ }^{2,3}$ The result can be decreased or absent vaginal lubrication in response to sexual stimulation, failure of normal genital engorgement, and delay or loss of orgasmic potential, all of which could seriously disrupt a woman's sexual life.

\section{The Sexual Ilistory}

For years the American Diabetes Association has recommended obtaining a thorough genitourinary history, including sexual history, whenever physicians examine patients with diabetes. ${ }^{4}$ As pointed out by the authors, the actual rates of compliance with this guideline are dismal. Why such poor history taking, especially when dealing with condi-

Submitted, revised, 29 January 1998.

From the Department of Family Practice, University of Washington School of Medicine, Seattle. Address reprint requests to Chris Herter, MD, Department of Family Practice, UWMC Roosevelt, Box 354775, Seattle, WA 98105. tions for which effective therapy is known to exist?

To begin with, family physicians can all agree that discussions of human sexuality are probably not comfortable for either the patient or health care provider. The data presented by Zweifler and colleagues $^{1}$ in this issue of the Fournal confirm that our patients are not likely to raise sexual issues on their own without some type of prompting.

Second, managed care has compressed the office visit in the name of efficiency, and taking a complete sexual history can be time consuming. In general terms most patients who express dissatisfaction regarding unmet expectations for care already cite too little time for history taking as the single most frequent cause of their concern. ${ }^{5}$ Pressured to see more patients in the same number of office hours, physicians are challenged simply to deal with the basics during interactions with patients.

Our patients' as well as our own discomfort with a potentially uncomfortable subject is the least difficult issue. As is the case with any skill, frequent application will ultimately lead to improved proficiency. This theory lies behind our training; physicians learn the art of taking a good general history by doing hundreds during medical and postgraduate education. We should make it a habit to include a thorough sexual history for all of our patients with diabetes during their annual examinations and at any time new physical complaints warrant.

Squeezing the time for a sexual history from our busy practices can be a greater challenge. Published research analyzing the effect of managed care on office hours and practices of the primary care provider is surprisingly scant considering the large volume of editorials and opinions on the subject. A review of the hundreds of articles written by angered and frustrated physicians makes apparent the widespread dissatisfaction that pervades all of medicine regarding managed care. Everyone feels overworked, pressed for time, and worried about the future. The effect on health care in general is not trivial. At least one well-considered article confirms a difference when comparing how primary 
care providers approach patients with managed care and how they approach patients with traditional medical insurance. ${ }^{6}$

As troubling as this development is, there is little argument that prepaid medicine has unquestionably changed the way we all look at health care, and many providers feel burdened by such strong emphasis on numbers and the bottom line. A more proactive look, however, can provide some insight. By addressing the issue of sexual functioning up front, we can actually save time spent in future office visits. After all, the cost effectiveness of practicing good, thorough, proactive medicine in all other areas of diabetes care has been well established. ${ }^{7}$ As providers become more skilled at obtaining information about sexual history, they will be able to get the clinically important data they need in a few extra minutes.

\section{Differential Diagnosis}

Sexual dysfunction in diabetes can result from damage to the autonomic nerves and small vessels that supply the genital region. In the assessment of sexual dysfunction, physicians must also consider behavioral as well as iatrogenic causes. As a rule psychologic and relationship issues are common causes of sexual difficulty, so it stands to reason that many of patients with diabetes could be spared an intensive organic workup if the clinician discovered a psychogenic cause early on. For example, if a patient has had diabetes for only a few years, has nearly normal glycemic control, and has no indication of autonomic or peripheral neuropathy on physical examination, diabetes-related sexual dysfunction would be very unlikely.

Similarly, although we all agree $\beta$-blockers are associated with sexual dysfunction in both men and women, it should be stressed that any antihypertensive regimen can cause sexual dysfunction, so carefully reviewing the medication list becomes critically important. A multitude of medications used to treat conditions relating to or coexistent with diabetes can also affect sexual functioning.

\section{Treatment}

\section{Male Dysfunction}

\section{Vacuum and Prostbetic Systems}

These systems provide the most basic way to generate an erection with tumescence sufficient for penetration and coital activity. The currently available devices use a cylindrical chamber that fits over the flaccid penis and against the body. Before use, a thick rubber band is stretched over the tube. Once the tube is positioned properly, an air pump evacuates the chamber. Blood from the body flows into the venous sinuses, producing an erection. The rubber band is then slipped off the tube onto the shaft of the penis. After the chamber is removed, the band maintains the erection by preventing backflow of blood. After sexual activity, the band is easily removed, returning the organ to its nonerect state.

Another approach is to replace the nonfunctional erectile tissue with a prosthetic implant. There are a variety of devices, but all require surgical placement. One study of 74 men who chose this option found that 89 percent were able to experience normal coitus, but nearly one quarter (23 percent) required at least one follow-up operation for complications. ${ }^{8}$ In another study only 52 of 80 (65 percent) men were able to return to normal sexual activity after implant surgery. ${ }^{9}$ For some patients, this type of therapy might be ideal; however, every candidate must be made aware of the possible complications and need for frequent follow-up.

\section{Vasoactive Drugs}

Several medical treatments have been used to augment male sexual function. Papaverine, prostaglandin $\mathrm{E}_{1}$, or alprostadil injected directly into the penis will produce an erection in most men. Various mixtures of these agents with other vasoactive compounds have also been successful, but all injection therapies have a high dropout rate. One study found that 56 percent of men who started intracavernosal injections had discontinued the treatment after only 1 year. ${ }^{10}$ Among the many reasons cited by the 100 participants for stopping therapy were desire for a permanent modality of therapy (29 percent), lack of a suitable partner (26 percent), fear of needles (23 percent), poor response (23 percent), fear of complications ( 22 percent), and lack of sexual spontaneity (21 percent).

Alprostadil, delivered transurethrally in the form of a small pellet, provides a way for men to avoid the discomfort of an injection. ${ }^{11}$ A special device is used to place the medicated pellet within the urethra. Despite the lack of a needle, there is still some discomfort, and recent data suggest that intraurethrally administered alprostadil is less efficacious than the more traditional therapies. ${ }^{12}$ 
Another alternative to standard treatments is sildenafil (Viagra), a drug that selectively inhibits the action of an isomer of phosphodiesterase found exclusively in the vascular cavernous tissue of the penis. ${ }^{13}$ When sildenafil is given systemically, its actions are limited to the corpus cavernosa, and an erection results. Because the drug is quickly absorbed and eliminated, there are few, if any side, effects. It can be administered orally before sexual activity. This drug could even prove useful in treating aspects of female sexual dysfunction related to diabetes.

\section{Female Dysfunction}

Female sexual dysfunction can sometimes be more subtle and difficult to recognize. Indeed, women with diabetes complain of a variety of symptoms, such as greater fatigue, diminished desire, and inadequate lubrication, as well as prolonged time to reach orgasm or the loss of orgasmic potential entirely. Family physicians should consider the entire spectrum of issues, and we should be frank in our discussions of the problem. For instance, orgasmic difficulties can stem from the dyspareunia of inadequate lubrication; recommending a safe lubricating product could be the best treatment. Anorgasmia could also result from changing physical needs for sexual stimulation, in which case recommending a thorough manual that discusses diversification of sexual behavior would be a better treatment. Again, it quickly becomes obvious that the sexual history provides the necessary information to formulate sound recommendations.

\section{Prevention}

Sexual dysfunction is a complication of diabetes. No studies to date have focused exclusively on what role glycemic control plays in the risk of developing sexual complications in diabetes. Nevertheless, neuropathic disease is undoubtedly the primary mediator of dysfunction in both women and men. The relation between glycemic control and the risk of neuropathy has been clearly established for type 1 diabetes in the Diabetes Control and Complications Trial. ${ }^{1+}$ Toyry and coworkers ${ }^{15}$ have found a link between diminished cardiovascular responses to deep breathing and orthostatic blood pressure challenges and poor glycemic control in 133 patients with type 2 diabetes. This finding represents a more than casual relation in both cases. Neuropathy can be prevented by glycemic control; therefore, sexual dysfunction, mediated by hyperglycemia in diabetes, can also be prevented.

\section{Summary}

Erectile dysfunction is more common in men with diabetes than in age-matched nondiabetic control groups. ${ }^{16}$ Women with diabetes probably have similar rates of dysfunction. The article by Zweifler et al $^{1}$ brings attention to a problem that primary care providers are uniquely qualified to handle. Because 90 percent of patients with diabetes are cared for by family physicians and primary care internists, ${ }^{17}$ they will be the first who diagnose any associated sexual dysfunction and should be the first to treat it. We are dealing with a disease for which natural history is now well known, and our goal, when possible, should be directed toward prevention.

\section{Christian D. Herter, MD University of Washington Seattle}

\section{References}

1. Zweifler J, Padilla A, Schafer S. Barriers to recognition of erectile dysfunction among diabetic MexicanAmerican men. J Am Board Fam Pract 1998;11: 259-63.

2. Dunning $P$. Sexuality and women with diabetes. $\mathrm{Pa}$ tient Educ Couns 1993;21(1-2):5-14.

3. Wincze JP, Albert A, Bansal S. Sexual arousal in diabetic females: physiological and self-report measures. Arch Sex Behav 1993;22:587-601.

4. American Diabetes Association. Clinical Practice Recommendations 1997. Diabetes Care 1997;20 (Suppl 1):S5-18.

5. Kravitz RL, Callahan EJ, Paterniti D, Antonius D, Dunham M, Lewis CE. Prevalence and sources of patients' unmet expectations for care. Ann Intern Med 1996;125:730-7.

6. Morgan RO, Virnig BA, DeVito CA, Persily NA. The Medicare-HMO revolving door-the healthy go in and the sick go out. N Engl J Med 1997; 337: 169-75.

7. Herman WH, Dasbach EJ, Songer TJ, Eastman $\mathrm{RC}$. The cost-effectiveness of intensive therapy for diabetes mellitus. Endocrinol Metab Clin North Am 1997;26:679-95.

8. Nukui F, Okamoto S, Nagata M, Kurokawa J, Fukui J. Complications and reimplantation of penile implants. Int J Urol 1997;4(1):52-4.

9. Rossi D, Ayuso D, Rattier C, Bladou F, I Iermanowicz M, Serment G. Clinical experience with 80 inflatable penile prostheses. Eur Urol 1997;31:335-8.

10. Sundaram CP, Thomas $W$, Pryor LE, Sidi $A \Lambda$, Billups K, Pryor JL. Long-term follow-up of pa- 
tients receiving injection therapy for erectile dysfunction. Urology 1997;49:932-5.

11. Padma-Nathan H, Hellstrom WJ, Kaiser FE, Labasky RF, Lue TF, Nolten WE, et al. Treatment of men with erectile dysfunction with transurethral alprostadil. Medicated Urethral System for Erection (MUSE) Study Group. N Engl J Med 1997; 336:1-7.

12. Werthman P, Rajfer J. MUSE therapy: preliminary clinical observations. Urology 1997;50:809-11.

13. Boolell M, Allen MJ, Ballard SA, Gepi-Attee S, Muirhead GJ, Naylor AM, et al. Sildenafil: an orally active type 5 cyclic GMP-specific phosphodiesterase inhibitor for the treatment of penile erectile dysfunction. Int J Impot Res 1996;8(2):47-52.
14. The effect of intensive treatment of diabetes on the development and progression of long-term complications in insulin-dependent diabetes mellitus. The Diabetes Control and Complications Trial Research Group. N Engl J Med 1993;329:977-86.

15. Toyry JP, Niskanen LK, Mantysaari MJ, Lansimies EA, Uusitupa MI. Occurrence, predictors, and clinical significance of autonomic neuropathy in NIDDM. Ten-year follow-up from the diagnosis. Diabetes 1996;45:308-15.

16. Hakim LS, Goldstein I. Diabetic sexual dysfunction. Endocrinol Metab Clin North Am 1996;25:379-400.

17. Harris MI, Eastman RC, Siebert C. The DCCT and medical care for diabetes in the US. Diabetes Care 1994;17:761-4. 